\title{
Determinação da carga de treino nos exercícios supino e rosca bíceps em mulheres jovens
}

\author{
Ronei Silveira Pinto \\ Daniel Félix \\ Eduardo Lusa Cadore \\ Marcelo Cardoso \\ Laboratório de Pesquisa do Exercício, Universidade Federal do Rio Grande do Sul, Porto \\ Alegre, RS, Brasil
}

Resumo: O objetivo deste estudo foi avaliar a força máxima dinâmica (1RM) nos exercícios supino e rosca scott, e relacioná-la com a massa corporal total (MCT) e com a massa corporal magra (MCM), bem como avaliar o número máximo de repetições em diferentes percentuais de 1RM. Onze mulheres (24 $\pm 1,4$ anos) foram submetidas à avaliação da composição corporal, testes de 1RM e testes de repetições máximas em $50,60,70$ e $80 \%$ de 1RM. No tratamento dos dados, foram utilizados a regressão linear múltipla, a ANOVA para medidas repetidas e o teste t pareado. A MCM é a variável que melhor explica a variância de 1RM, podendo ser utilizada para a determinação de um coeficiente para a estimativa da carga de treino. Houve diferenças significativas entre os números de repetições em todos os percentuais e em ambos os exercícios [(supino $(p=0,000)$ e rosca scott $(p=0,000)]$, sendo esses números progressivamente menores com o incremento da carga.

Palavras-chave: Musculação. Força muscular. Eletromiografia.

\section{Defining the training load in bench press and biceps curl exercises in young women}

\begin{abstract}
The purpose of this study was to assess maximum strength (1RM) in the bench press and biceps curl exercises, and relate it to total body mass (BM) and fat-free mass (FFM), as well as to assess the maximum repetition number at different percentages of 1-RM. Eleven women (age: $24 \pm 1.4$ years) were submitted to body composition assessment, 1-RM tests, and maximum repetition tests at 50,60,70 and $80 \%$ of 1 -RM. For data treatment, multiple linear regression, repeated measures ANOVA and paired t-test were used. FFM explains better than BM the 1-RM variance and can be used to determine a coefficient to estimate the training load. Significant differences were found in the maximum repetition numbers in all percentages and both exercises (bench press $(p=0,000)$ and biceps curl $(p=0,000)$. These results revealed a gradual decrease in the number of repetitions as the load increased.
\end{abstract}

Key Words: Resistance Training. Muscle Strength. Eletromiography.

\section{Introdução}

Atualmente, o treinamento de força é uma das formas mais populares de exercício para melhora da aptidão física (COTTERMAN et al., 2005), pois entre seus benefícios estão incluídos incrementos da força, da potência, do tamanho e resistência muscular (SAKAMOTO; SINCLAIR, 2006), da massa óssea, da espessura de tecidos conectivos e redução da gordura corporal total, além da prevenção e manejo de diversas condições patológicas e da saúde em geral (WOLFE et al., 2004; BIRD et al., 2005).

No entanto, para que estes benefícios sejam alcançados, alguns pontos devem ser considerados, entre os quais a individualização do programa de treino, a progressão e a manutenção sobrecarga, além das variáveis agudas do treinamento de força. Estas últimas foram identificadas pela primeira vez por Kraemer
(1983) e são constituídas pela seleção dos exercícios, ordem dos exercícios, número de séries, intensidade (carga utilizada), duração do período de repouso, velocidade e frequência de treino (KRAEMER, 1983; SPREUWENBERG et al., 2006; FLECK e KRAEMER, 2006; ACSM, 2009). O controle destas variáveis determinará o nível de adaptação dos sistemas neuromuscular, neuroendócrino e músculo-esquelético, quer de forma aguda quer crônica (BIRD et al., 2005), e este controle deve estar de acordo com os objetivos do treino a ser executado (SAKAMOTO; SINCLAIR, 2006).

Dentre as variáveis agudas acima referidas, a intensidade (carga utilizada) é considerada por alguns autores como a mais importante de ser controlada na elaboração de um programa de treino de força (FLECK; KRAEMER, 2006; KRAEMER, 2003), pois esta delimitará o número 
de repetições possíveis de serem realizadas e o tempo de recuperação necessário entre as séries e exercícios (BAECHLE; EARLE, 2008) e, por consequência, determinará os efeitos do treinamento sobre o desenvolvimento da força (HATFIELD et al., 2006) e as adaptações fisiológicas dele decorrentes (DOURIS et al., 2006).

Segundo Fleck e Kraemer (2006), a intensidade pode ser determinada a partir do teste direto de uma repetição máxima (1RM), ou por fórmulas preditivas derivadas de testes submáximos, em que a utilização de coeficientes específicos de aproximação deste valor facilita e reduz o seu tempo de execução. Nesta perspectiva de utilização, após a definição do valor de 1RM em determinado exercício, a prescrição da carga de treino é determinada por percentuais deste valor (\%1RM), a qual deve estar de acordo com os objetivos pretendidos. A intensidade pode ainda ser definida a partir do Índice de Percepção do Esforço (TIGGEMANN et al., 2010).

A maioria das formas anteriormente citadas envolve testes que podem ser mais ou menos complexos, sendo que, segundo Sakamoto e Sinclair (2006), o teste de 1RM pode estar associado a lesões quando executado incorretamente ou exigir muito tempo para a sua execução, sobretudo em sujeitos sem experiência com exercícios de força (ABADIE; WENTWORTH, 2000). Ao contrário, Reynolds et al. (2006) afirmam que o uso do teste de 1RM parece ser um método seguro de avaliação da força em indivíduos treinados e destreinados, apesar de o considerarem contra-indicado para algumas populações.

O uso de coeficientes para a predição de 1RM evita a realização de testes máximos e reduz o tempo destinado à determinação da carga de treino, embora seja de natureza preditiva. A utilização destes coeficientes foi inicialmente proposta por Baechle e Groves (2000), sendo constituídos para homens e mulheres a partir da relação entre valores de $1 \mathrm{RM}$ e a massa corporal total (MCT) em exercícios de força específicos. Estes coeficientes podem ainda levar em conta a massa corporal magra (MCM), uma vez que esta estrutura biológica é a maior responsável pela produção de força (COTTERMAN et al., 2005).

Analisando o que foi exposto, percebe-se que a determinação da carga de treino constitui-se numa tarefa complexa e que a utilização de coeficientes talvez possa reduzir o tempo destinado à predição de $1 \mathrm{RM}$ e também o risco de lesões decorrentes de um teste máximo. No entanto, somente a determinação do valor de 1RM parece não ser suficiente para a determinação da carga de treino, uma vez que o número de repetições máximas (RMs) em determinados percentuais deste valor (\% de 1RM) varia de um exercício de força para outro (SAKAMOTO; SINCLAIR, 2006; HOEGER et al., 1987; HOEGER et al., 1990). Portanto, a determinação desta relação (\% de $1 \mathrm{RM} \times \mathrm{RMs}$ possíveis) em cada exercício de força torna-se necessária.

Sendo assim, o objetivo deste estudo experimental foi determinar coeficientes para a predição de 1RM e avaliar a relação deste valor estimado com as massas total (MCT) e magra (MCM). Além disso, pretendeu-se avaliar a relação entre percentuais de $1 \mathrm{RM}$ e o número de repetições máximas (RMs) em mulheres jovens sem experiência em treinamento de força nos exercícios supino e rosca scott, ambos com pesos livres.

\section{Material e Métodos Grupo experimental}

O grupo experimental foi composto por 11 mulheres (24 $\pm 1,4$ anos), selecionadas de forma intencional a partir dos seguintes critérios: apresentar Índice de Massa Corporal (IMC) considerado normal ou ideal, entre 18,5 e 25 $\mathrm{kg} / \mathrm{m}^{2}$ (OPAS/OMS BRASIL, 2003); não apresentar limitações físicas ou problemas músculo-esqueléticos que pudessem afetar os testes; não estarem envolvidas com prática de exercícios de força por, pelo menos, seis meses antes do início do estudo. As características da amostra são apresentadas abaixo na Tabela 1 . Os participantes foram informados previamente sobre os protocolos e procedimentos da pesquisa e assinaram um termo de consentimento informado para participarem do estudo, de acordo com as exigências do Comitê de Ética da UFRGS (Protocolo №: 19039).

Tabela 1- Caracterização do grupo experimental $(n=11)$

\begin{tabular}{ccc}
\hline Características & Média & Desvio Padrão \\
\hline Idade $(\mathrm{anos})$ & 24 & 1,42 \\
Massa $(\mathrm{kg})$ & 58,50 & 6,52 \\
Estatura $(\mathrm{m})$ & 1,64 & 0,69 \\
IMC $\left(\mathrm{kg} / \mathrm{m}^{2}\right)$ & 21,79 & 1,62 \\
\hline
\end{tabular}




\section{Equipamentos}

Para as medidas de avaliação antropométrica e de composição corporal foram utilizados um estadiômetro de metal da marca FILIZOLA, com resolução de $1 \mathrm{~cm}$, uma balança analógica da marca FILIZOLA, com resolução de $0,1 \mathrm{~kg}$ e um compasso para dobras cutâneas da marca LANGE, com resolução de $1 \mathrm{~mm}$. Já para os testes de força máxima (1RM) foram utilizadas anilhas de 1, 2, 3, 5 e $10 \mathrm{~kg}$, barra longa de $10 \mathrm{~kg}$ com grampos (para o supino), barra curta de $6 \mathrm{~kg}$ com grampos (para o rosca scott), banco de supino plano e banco scott (ambos da marca
World Sculptor), sendo este último com inclinação de 30 graus. Um metrônomo da marca QUARTZ foi utilizado para determinar o ritmo de execução das repetições máximas (RMs).

\section{Procedimentos}

Para a realização dos testes foram necessárias seis sessões, sendo a primeira destinada à avaliação da composição corporal e familiarização dos exercícios, a segunda para a realização do teste de $1 \mathrm{RM}$ dos exercícios determinados, e as quatro sessões restantes para os testes de RMs nos percentuais 50, 60, 70 e $80 \%$ de $1 \mathrm{RM}$, ordenados aleatoriamente. A figura 1 apresenta de modo simplificado a organização das sessões de avaliação e de testes.

Figura. 1- Desenho Experimental Simplificado

\begin{tabular}{|c|c|c|c|c|c|c|c|c|c|c|}
\hline Sessão 1 & $\begin{array}{l}\frac{0}{N} \\
\frac{2}{d} \\
\text { I্ }\end{array}$ & Sessão 2 & $\begin{array}{l}\frac{0}{N 0} \\
\frac{1}{d} \\
\text { 은 }\end{array}$ & Sessão 3 & $\begin{array}{l}\frac{0}{\pi} \\
\frac{1}{d} \\
\stackrel{d}{\leq}\end{array}$ & Sessão 4 & $\begin{array}{l}\frac{0}{\text { N }} \\
\frac{1}{d} \\
\text { 咅 }\end{array}$ & Sessão 5 & $\begin{array}{l}\frac{0}{N \pi} \\
\frac{1}{d} \\
\text { I্ }\end{array}$ & Sessão 6 \\
\hline $\begin{array}{l}\text { Avaliação: } \\
\text { composição } \\
\text { corporal e nível } \\
\text { de atividade } \\
\text { física, além de } \\
\text { familiarização }\end{array}$ & 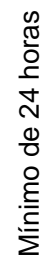 & $\begin{array}{l}\text { Teste de } \\
\text { 1RM }\end{array}$ & 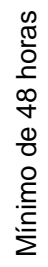 & $\begin{array}{c}\text { Teste de } \\
\text { repetiçõe } \\
s \\
\text { máximas }\end{array}$ & 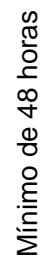 & $\begin{array}{l}\text { Teste de } \\
\text { repetições } \\
\text { máximas }\end{array}$ & 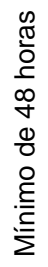 & $\begin{array}{c}\text { Teste de } \\
\text { repetiçõe } \\
s \\
\text { máximas }\end{array}$ & 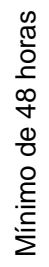 & $\begin{array}{c}\text { Teste de } \\
\text { repetições } \\
\text { máximas }\end{array}$ \\
\hline
\end{tabular}

\section{Protocolo para avaliação da composição corporal}

Para a avaliação da composição corporal foram feitas as medidas de estatura (em metros) e da massa corporal (em quilogramas). Posteriormente, foram feitas as medidas de sete dobras cutâneas: triciptal, subescapular, suprailíaca, abdominal, peitoral, axilar média e coxa (JACKSON et al.,1980). A partir deste protocolo obteve-se a densidade corporal que foi aplicada na fórmula de Siri (1993) para a obtenção do percentual de gordura. Pelo percentual de gordura calculou-se a massa gorda (\%G x massa corporal/100) e, posteriormente, a massa magra (MCM) (massa corporal - massa gorda).

\section{Protocolo para os testes de 1RM}

Os testes de 1RM foram realizados em uma única sessão, após outra de familiarização, em que foram definidos o ritmo de execução (definido por metrônomo) e a técnica de execução dos exercícios testados. A familiarização foi realizada para minimizar os efeitos da aprendizagem do exercício para o teste de 1RM (HATFIELD et al., 2006). O 1RM foi determinado por tentativa e erro, tendo sido utilizada a tabela de Lombardi (1989) para estimar a carga em cada tentativa. 0 intervalo entre as tentativas foi de no mínimo 5 minutos (SAKAMOTO; SINCLAIR, 2006; KRAEMER, 2003; REYNOLDS et al., 2006). O $1 \mathrm{RM}$ foi determinado em apenas uma sessão de teste, não tendo sido reconfirmado em outra sessão, constituindo-se assim em uma possível limitação metodológica do estudo.

Para o exercício supino a largura de empunhadura da barra foi estabelecida como sendo levemente mais afastada $(\sim 15 \mathrm{~cm})$ que a largura dos ombros (SAKAMOTO; SINCLAIR, 2006; ABADIE; WENTWORTH, 2000). Os participantes deviam manter suas costas sobre 0 banco e os pés sobre um apoio na base deste durante todo o exercício. Além disso, foi dada assistência para retirar a barra do suporte (COTTERMAN et al., 2005; BAECHLE; GROVES, 2000). A barra deveria ser baixada até tocar levemente a parte anterior do tórax (esterno), e então empurrada para cima até a total extensão 
dos cotovelos em cada repetição, sem hiperextensão dos cotovelos. Não foi permitido o rebote exagerado da barra contra o tórax e o arqueamento da coluna lombar (SAKAMOTO; SINCLAIR, 2006). Para o exercício rosca scott, os indivíduos deviam manter uma posição sentada com a região anterior do tórax encostada na almofada, sendo a largura de empunhadura da barra semelhante à largura dos ombros. No início do teste, a barra era retirada do suporte pelos avaliadores, para, depois disso, serem realizadas as fases concêntrica e excêntrica do movimento, esta última sem a hiperextensão dos cotovelos (BAECHLE; GROVES, 2000). Durante cada tentativa e para cada repetição em ambos os exercícios foi dado encorajamento verbal aos indivíduos (SAKAMOTO; SINCLAIR, 2006).

\section{Cálculo dos Coeficientes}

Os coeficientes individuais foram calculados a partir do valor de 1RM em cada exercício dividido pela MCT (1RM/MCT) e pela MCM (1RM/MCM).

\section{Protocolo para os testes de percentuais de $1 R M$}

Os testes de percentuais de 1RM foram realizados em dias diferentes. Foi testado 0 número de RMs nos percentuais de 50, 60, 70 e $80 \%$ do $1 \mathrm{RM}$ avaliado. Estes percentuais foram ordenados aleatoriamente por meio de sorteio para cada um dos participantes. Foi usada a randomização para eliminar os efeitos da ordem sobre os resultados (HATFIELD et al., 2006). Entre a sessão de testes de 1RM e entre cada uma das sessões de testes de percentuais de $1 \mathrm{RM}(50,60,70,80 \%)$ foi dado um intervalo de pelo menos 48 horas (SAKAMOTO; SINCLAIR, 2006; SPREUWENBERG et al., 2006; ABADIE; WENTWORTH, 2000; REYNOLDS et al., 2006). Os indivíduos executaram cada exercício até a falha concêntrica com o ritmo de execução determinado por um metrônomo, sendo utilizada a cadência de 60 bpm com dois batimentos para cada fase, ou seja, 2 segundos para a fase concêntrica e 2 segundos para a fase excêntrica do movimento (ABADIE; WENTWORTH, 2000), não sendo permitidas pausas entre as fases de contração.

\section{Análise estatística}

O modelo de regressão linear múltipla foi usado em ambos os exercícios para determinar qual das variáveis independentes (massa corpora total - MCT e massa magra - MCM) explicaria melhor o comportamento da variável dependente (1RM). Para a comparação entre os coeficientes obtidos a partir das MCT e MCM foi utilizado um teste t de Student pareado. Na comparação do número de repetições máximas (RMs) atingido nos diferentes percentuais em cada exercício utilizamos o teste de ANOVA para medidas repetidas e, o teste $\mathrm{t}$ de Student pareado para comparação entre os exercícios em cada percentual adotado. Para relacionar o percentual de 1RM com o número RMs em ambos os exercícios foi usada uma regressão linear. Foi adotado um nível de significância $p \leq 0,05$ e o pacote estatístico utilizado foi o SPSS versão 15.0 e o Microsoft Excel 2003, ambos para Windows.

\section{Resultados}

\section{Valores de $1 R M$ nos exercícios supino e rosca scott}

Os valores relativos ao $1 \mathrm{RM}$ (média $\pm \mathrm{DP}$ ) nos exercícios supino e rosca scott estão apresentados na tabela 2. Os coeficientes gerados a partir da relação $1 \mathrm{RM} / \mathrm{MCT}$ foram de 0,47 para o supino e de 0,22 para o rosca scott e a média dos coeficientes gerados a partir da relação $1 \mathrm{RM} / \mathrm{MCM}$ foi de 0,65 para o supino e de 0,30 para o rosca scott.

Tabela 2- Valores de MCT, MCM, bem como de $1 \mathrm{RM}$ e Coeficientes relativos à MCT e MCM, nos exercícios supino e rosca scott $(\mathrm{n}=11)$.

\begin{tabular}{lcccc}
\hline & \multicolumn{2}{c}{ Supino } & \multicolumn{2}{c}{ Rosca Scott } \\
\hline 1RM (kg) & Média & Desvio Padrão & Média & Desvio Padrão \\
MCT (kg) & 27,09 & 2,91 & 12,45 & 1,62 \\
MCM (kg) & 58,50 & 6,52 & 58,50 & 6,52 \\
Coeficiente 1RM / MCT & 41,85 & 3,08 & 41,85 & 3,08 \\
Coeficiente 1RM / MCM & 0,47 & 0,06 & 0,22 & 0,03 \\
a & $0,65^{\text {a }}$ & 0,07 & $0,30^{\text {a }}$ & 0,04 \\
\hline Diferenças significativas entre os coeficientes gerados a partir & da MCM e da MCT $(\mathrm{p}=0,000)$.
\end{tabular}


Correlação entre os valores de 1 RM e as massas corporal total (MCT) e magra (MCM)

Tanto a MCT quanto a MCM não apresentaram correlação significativa com o 1RM para os dois exercícios avaliados. Na tabela 3 , a seguir, são apresentados os valores de correlação, a significância e o coeficiente de determinação para ambos os exercícios.

Tabela 3- Valores de correlação entre 1RM e MCM e MCT no supino e rosca scott $(n=11)$.

\begin{tabular}{ccccccc}
\hline & \multicolumn{3}{c}{ Supino } & \multicolumn{3}{c}{ Rosca Scott } \\
\hline & $\mathbf{r}$ & sig. & $\mathbf{R}^{\mathbf{2}}$ & $\mathbf{r}$ & sig. & $\mathbf{R}^{\mathbf{2}}$ \\
\hline 1RM x MCT & 0,159 & 0,641 & 0,03 & 0,085 & 0,803 & 0,01 \\
1RM x MCM & 0,281 & 0,403 & 0,08 & 0,379 & 0,251 & 0,14 \\
\hline
\end{tabular}

\section{Comparação entre coeficientes}

Foi encontrada uma correlação entre o coeficiente obtido pela MCT e aquele obtido pela MCM no exercício supino $(r=0,92)$ e no exercício rosca scott $(r=0,95)$ e esta foi estatisticamente significativa em ambos os exercícios $(p=0,000)$.
No entanto, um teste $\mathrm{t}$ pareado mostrou que houve diferenças estatisticamente significativas entre os coeficientes, sendo que os índices médios apresentados em relação à MCM foram sempre superiores àqueles apresentados pela $\operatorname{MCT}(p=0,000)$.

Figura 2- Diferença entre o 1RM avaliado ( - --- ) e o 1RM predito por coeficientes gerados pela MCT ( - - ) e pela MCM $(----)$, no supino

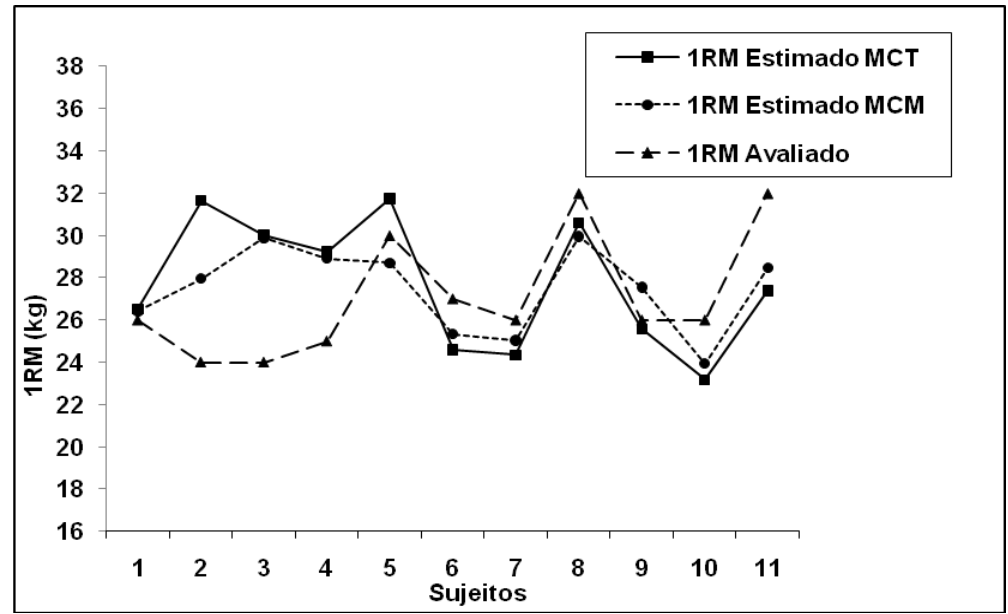

Figura 3. Diferença entre o 1RM avaliado ( --- ) e o 1RM predito por coeficientes gerados pela MCT ( --$)$ e pela MCM (-----), no rosca scott

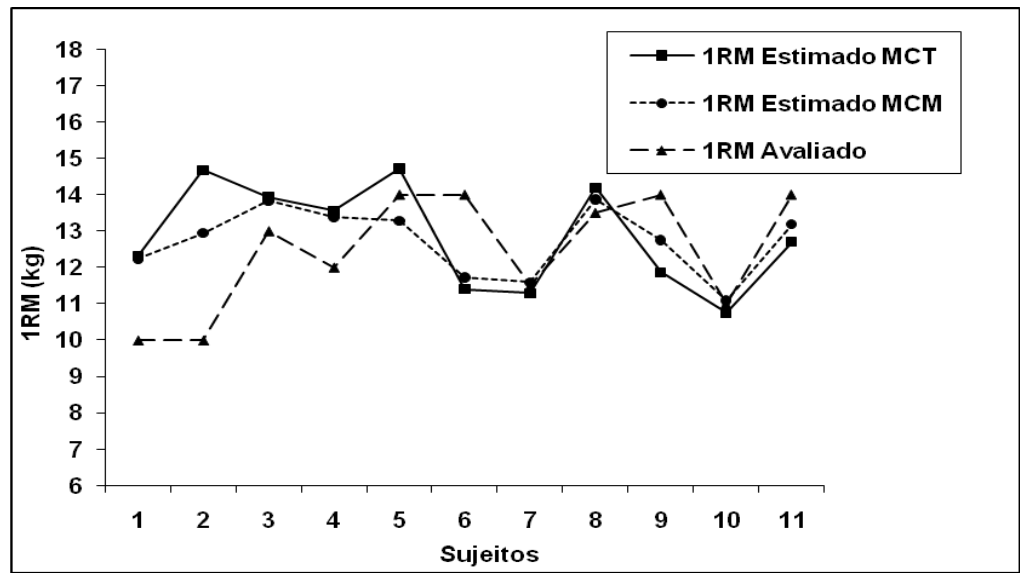

A média dos coeficientes gerados a partir da relação $1 \mathrm{RM} / \mathrm{MCT}$ e $1 \mathrm{RM} / \mathrm{MCM}$ foi de 0,47 e 0,65 no exercício supino, e de 0,22 e 0,30 no exercício rosca scott, respectivamente. Quando usados para estimar o 1RM no exercício supino, estes coeficientes médios apresentaram diferentes correlações com esta variável, sendo que o coeficiente gerado pela $\mathrm{MCM} \quad\left(\mathrm{R}^{2}=0,56\right)$ demonstrou uma correlação superior àquela 
apresentada pelo coeficiente gerado pela MCT $\left(R^{2}=0,36\right)$. A seguir, a figura 2 exibe a diferença entre $\circ 1 \mathrm{RM}$ avaliado e o $1 \mathrm{RM}$ predito a partir destes coeficientes para o exercício supino.

No exercício rosca scott, estes coeficientes médios também apresentaram diferentes correlações com esta variável, sendo que 0 coeficiente gerado pela MCM $\left(R^{2}=0,68\right)$ mostrou igualmente uma correlação superior àquela apresentada pelo coeficiente gerado pela MCT $\left(R^{2}=0,53\right)$. Abaixo, a figura 3 mostra a diferença entre $\circ 1 \mathrm{RM}$ avaliado e $\circ 1 \mathrm{RM}$ predito a partir destes coeficientes para o exercício rosca scott.

\section{Número de repetições máximas (RMs)}

Nos exercícios supino e rosca scott o número de RMs atingido em cada percentual decresceu conforme o aumento deste percentual, mostrando que conforme a carga é incrementada há uma evidente diminuição no número de repetições realizadas. Houve diferenças estatisticamente significativas $(p<0,001)$ entre os percentuais de 1 RM avaliados $(50,60,70$, e $80 \%)$ para ambos os exercícios. A tabela 4 apresenta o número de RMs (média e desvio padrão) obtidos em cada percentual de 1RM testado para cada exercício.

Tabela 4- Número de repetições máximas (RMs) obtido nos exercícios supino e rosca scott $(\mathrm{n}=11)$

\begin{tabular}{|c|c|c|c|c|}
\hline & \multicolumn{4}{|c|}{ Intensidade de 1RM } \\
\hline & $50 \%$ & $60 \%$ & $70 \%$ & $80 \%$ \\
\hline \multicolumn{5}{|l|}{ Supino } \\
\hline Média & $22,82^{a b}$ & $17,55^{\mathrm{ab}}$ & $10,73^{a b}$ & $6,00^{a b}$ \\
\hline Desvio Padrão & 3,34 & 3,50 & 2,05 & 1,10 \\
\hline \multicolumn{5}{|l|}{ Rosca Scott } \\
\hline Média & $50,55^{a b}$ & $32,27^{a b}$ & $17,36^{a b}$ & $9,09^{a b}$ \\
\hline Desvio Padrão & 12,07 & 9,62 & 3,72 & 2,55 \\
\hline
\end{tabular}

As figuras 4 e 5 abaixo, referentes aos exercícios supino e rosca scott respectivamente, mostram a dispersão dos valores nos diferentes percentuais avaliados $(50,60,70$, e $80 \%)$, sendo possível perceber que esta dispersão reduz-se à medida que o percentual aumenta em ambos os exercícios.

Figura 4- Dispersão dos valores no supino nos diferentes percentuais de 1RM avaliados

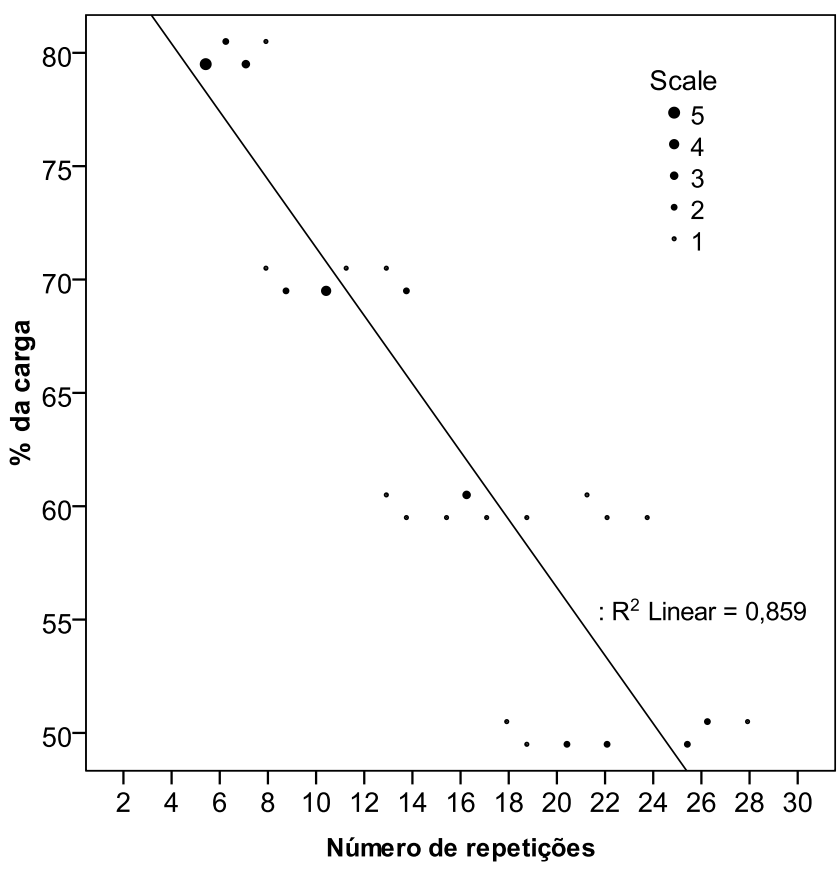


Comparação entre o número de repetições máximas (RMs) obtido em cada exercício

Houve diferenças estatisticamente significativas $(p<0,000)$ entre cada um dos percentuais avaliados $(50,60,70$, e $80 \%$ de $1 \mathrm{RM})$ em ambos os exercícios, sendo encontrado um número de RMs superior no exercício de rosca bíceps scott em todos estes percentuais quando comparado ao exercício supino. Também foi possível observar que o decréscimo no número de RMs foi superior no exercício rosca scott, ou seja, o delta percentual (diferença \% entre o máximo e o mínimo de repetições) foi mais expressivo no exercício rosca scott $(\Delta \%=82 \%)$ do que no exercício supino $(\Delta \%=73 \%)$.

Figura 5. Dispersão dos valores no rosca scott nos diferentes percentuais de 1RM avaliados

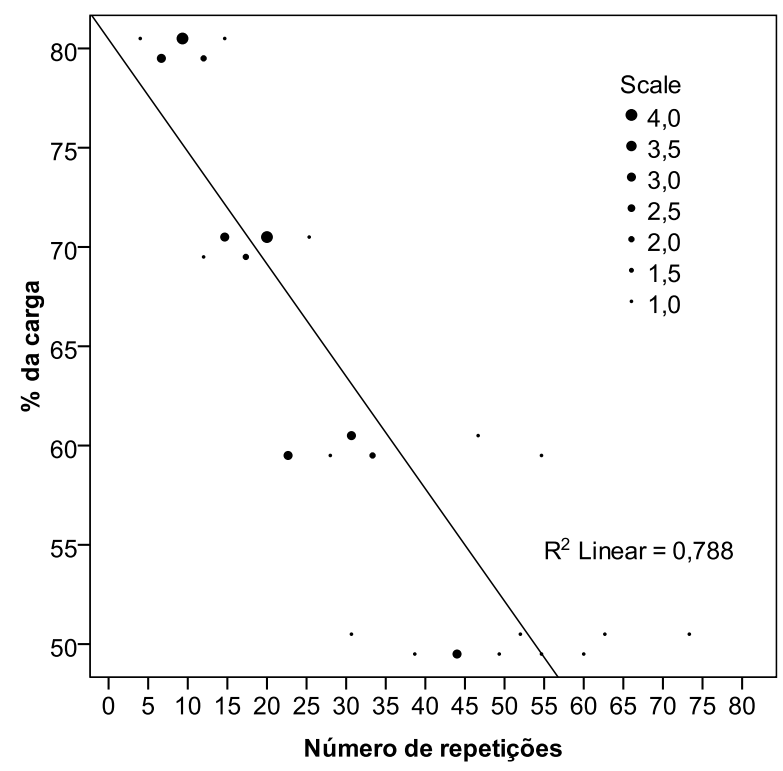

\section{Equações para a predição do \% de 1RM}

Uma regressão linear foi usada em ambos os exercícios para relacionar o percentual de 1RM com o número de repetições atingido. Com a utilização desta estratégia, foi gerada uma equação específica para cada exercício para estimar o percentual de 1RM a partir do número de repetições obtido.

No exercício supino a correlação entre o número de repetições e o percentual de carga foi elevada $\left(R^{2}=\right.$ 0,88), e a seguinte equação foi gerada:

$$
\rightarrow \% \text { do } 1 \mathrm{RM}=-1,9825 \times \text { número de } \mathrm{RMs}+95,033
$$

A seguir, a figura 6 mostra a relação entre o percentual de 1RM e o número de repetições máximas atingido em cada um destes percentuais no exercício supino.

Figura 6. Relação entre o \% de 1RM e o número de RMs atingido no supino

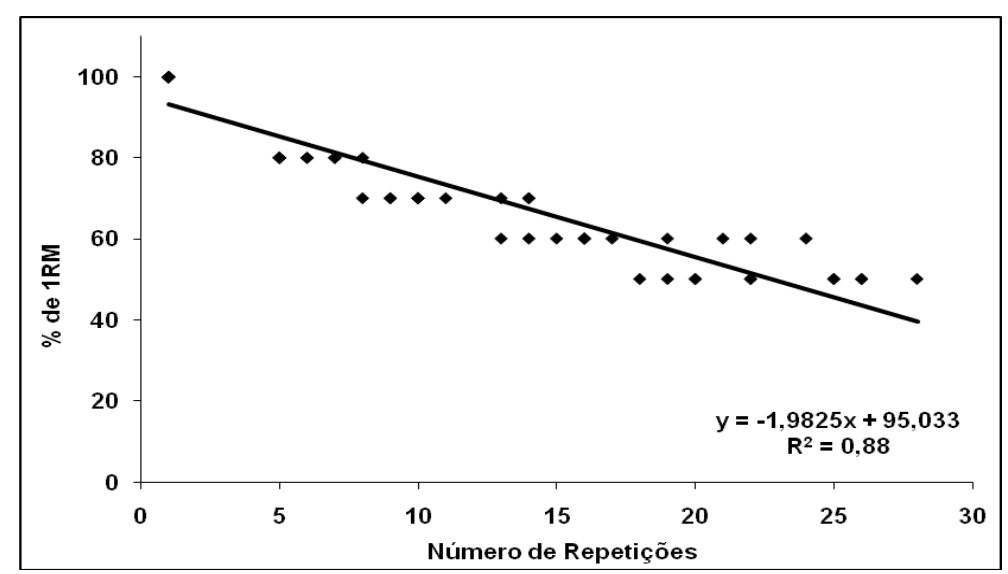


No exercício rosca scott a correlação entre o número de repetições e o percentual de 1RM também foi elevada $\left(R^{2}=0,78\right)$, e a seguinte equação foi gerada:

$$
\rightarrow \% \text { do } 1 \mathrm{RM}=-0,8031 \times \text { número de } \mathrm{RMs}+89,713
$$

Abaixo, a figura 7 mostra a relação entre o percentual de $1 \mathrm{RM}$ e o número de repetições máximas atingido em cada um destes percentuais no exercício rosca scott.

Figura 7. Relação entre o \% de 1RM e o número RMs atingido no rosca scott.

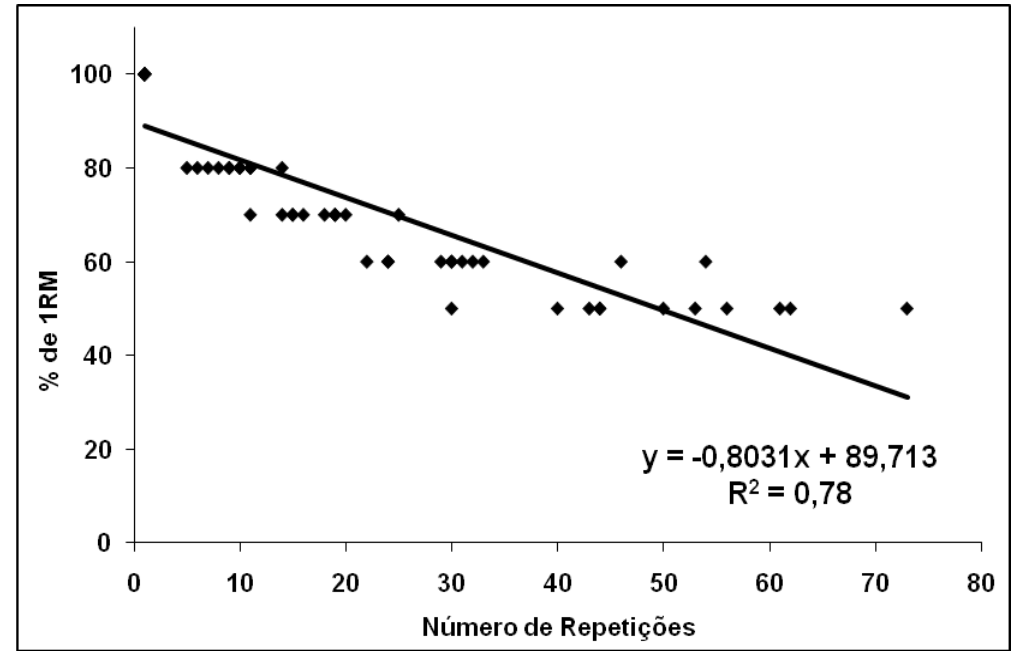

\section{Discussão}

Os testes de 1RM realizados neste estudo mostraram que os valores médios de força máxima apresentados no supino foram maiores que no rosca scott, e isto se deve ao fato da diferença no volume dos músculos ativados em cada um destes exercícios (flexores horizontais do ombro/extensores de cotovelo, e flexores de cotovelo, respectivamente). A produção de força muscular parece ser fortemente associada ao volume dos músculos ativados em condições máximas (FUKUNAGA et al., 2001).

Das relações entre 1RM e MCT, e 1RM e MCM pôde-se observar que ambas não demonstraram uma alta correlação com o 1RM, resultado semelhante ao encontrado no estudo de Westphal et al. (2006). Entretanto, entre as duas variáveis associadas ao $1 \mathrm{RM}$, aquela que melhor explicou a sua variância foi a MCM, possivelmente devido ao fato desta variável incluir a estrutura celular mais envolvida na produção de força, ou seja, a massa muscular, ao passo que a MCT inclui a massa de gordura, estrutura celular não relacionada à produção de força. Ainda se deve considerar que a amostra utilizada possuía um IMC abaixo de $25 \mathrm{~kg} / \mathrm{m}^{2}$, e que, portanto, não apresentava um elevado percentual de gordura, fazendo com que a diferença entre a MCT e a
MCM fosse reduzida. Pode-se especular que indivíduos que apresentam IMC e percentual de gordura mais elevados tenham esta diferença mais acentuada.

Os dois coeficientes gerados (a partir de MCT e MCM) em cada exercício apresentaram o mesmo comportamento e uma alta correlação entre $\mathrm{si}$, sendo que, àquele gerado pela $\mathrm{MCM}$ apresentou sempre uma predição mais acertada em relação àquele gerado pela $\mathrm{MCT}$, embora ambos não tenham obtido grande exatidão durante esta predição. Ainda assim, cabe ressaltar que a diferença média entre o 1RM predito pela MCM e o $1 \mathrm{RM}$ avaliado foi inferior a $10 \%$, valor pouco expressivo para sujeitos que se encontram na fase inicial de um programa de treino.

Embora alguns autores (DOURIS et al., 2006; REYNOLDS et al., 2006) não tenham encontrado uma relação significativa entre variáveis antropométricas, incluindo a massa corporal e a massa magra, e a força de 1RM, outros como Cotterman et al. (2005), referem que a composição corporal é também um dos fatores que afeta a produção de força, fato que está de acordo com os resultados observados no presente estudo. 
Sendo assim, os resultados do presente estudo permitem a proposição da utilização de coeficientes específicos relativos à MCM para a predição de 1RM nos exercícios supino e rosca $s c o t t$, sendo esta uma opção metodológica oposta à execução de testes diretos de $1 \mathrm{RM}$, sobretudo por serem testes contra-indicados para iniciantes e indivíduos sem experiência em treino de força (SAKAMOTO; SINCLAIR, 2006; BAECHLE; GROVES, 2000; ABADIE; WENTWORTH, 2000; BRZYCKI, 1993). Na hipótese dos testes de 1RM serem implementados, os coeficientes permitem ainda a determinação adequada da carga a ser inicialmente testada, o que minimiza o número de tentativas para a definição do 1RM final. Porém, deve-se salientar que os coeficientes são específicos para os exercícios e população avaliados.

Relativamente ao número de RMs realizado nos exercícios supino e rosca scott em cada percentual testado $(50,60,70$ e $80 \%)$, este decresceu conforme o aumento do percentual de $1 \mathrm{RM}$, fato também referido por outros investigadores (KRAEMER, 2003; HOEGER et al.,1987; HOEGER et al., 1990; PEREIRA; GOMES, 2007). Nos estudos clássicos de Hoeger et al. (1987) e Hoeger et al. (1990) é referida uma relação inversamente proporcional entre intensidade (carga) e RMs em vários exercícios de força, sendo esta diferente entre os exercícios avaliados. Esta relação foi identificada por Brzycki (1993) em vários exercícios de força como quase linear, sobretudo na faixa de 1-10 RMs.

No presente estudo, foi encontrado um número de RMs superior no exercício rosca scott em todos os percentuais testados (Tabela 4), quando comparado ao exercício supino, sendo que a relação entre RMs e os percentuais de 1 RM avaliados apresentou um comportamento diferente nestes exercícios. No exercício rosca scott ocorreu um acréscimo mais acentuado do número de RMs conforme o percentual de $1 \mathrm{RM}$ diminuiu (Figura 7), diferentemente do observado no exercício supino (Figura 6). A explicação para estes fatos não parece simples, e vários fatores podem estar a eles associados, entre os quais 0 tipo de equipamento, o tipo predominante de fibra muscular, o volume muscular, a relação forçavelocidade, a composição corporal, entre outros (COTTERMAN et al., 2005).

Tem sido referido na literatura que exercícios de força que ativam grandes grupos musculares (multiarticulares) suportam um número maior de RMs para um determinado percentual de $1 \mathrm{RM}$ em comparação a exercícios que ativam pequenos grupos musculares (monoarticulares) (KRAEMER; HAKKINEN, 2004), fato não observado nesta investigação. Uma hipótese que possivelmente explique os resultados encontrados na presente investigação é que em exercícios multiarticulares, como o supino, é exigida uma maior coordenação motora para a sua realização do que em exercícios monoarticulares (neste estudo representado pela rosca $S c o t t)$, em que o movimento é isolado em uma articulação e, como nesta investigação, realizado com significativa estabilidade articular. Assim, a inexperiência dos sujeitos avaliados com a prática de exercícios de força e a exigência coordenativa dos exercícios podem ter afetado os resultados.

A dispersão no número de RMs atingido em um mesmo percentual de $1 \mathrm{RM}$ pode também ser explicada, segundo alguns autores, pela composição muscular, sobretudo no que diz respeito à predominância de fibras do tipo I ou II. Douris et. al (2006) propõem que indivíduos com maior composição de fibras de tipo II mostram sinais de fadiga mais precoce e realizam um menor número de RMs do que àqueles que apresentam maior percentual de fibras de tipo I nos músculos avaliados, fato este também referido por Badillo e Ayestaran (2007). O maior número de RMs observado em todos os percentuais de $1 \mathrm{RM}$ no exercício rosca scott da presente investigação talvez possa ser explicado pela concentração paritária de fibras de tipo I e IIX no músculo bíceps braquial $(50 \%$ vs $50 \%$, respectivamente), relativamente aos músculos peitoral maior e tríceps braquial ( $42 \%$ vs $58 \%$, e $33 \%$ vs $67 \%$, respectivamente) (JOHNSON et al., 1973), principais músculos ativados nos exercícios rosca scott e supino, respectivamente.

As equações específicas geradas para estimar - percentual de 1RM pelo número de RMs realizadas nos exercícios supino e rosca scott sinalizam diferentes valores preditos. A partir das equações geradas neste estudo tem-se, por exemplo, 15RMs representando para o exercício supino $65,3 \%$ de $1 \mathrm{RM}$, enquanto que para o rosca scott estas mesmas 15RMs representariam $77,7 \%$ de $1 \mathrm{RM}$, ou seja, uma diferença de $12,4 \%$ entre os exercícios. Este fato claramente evidencia a limitação das equações de estimativa de 1RM a partir de uma única fórmula 
(LOMBARDI, 1989; BRZYCKI,1993; MAYHEW et al. 2008).

Outra importante consideração relativa às equações de predição de 1RM a partir do número de RMs executadas é que estas são tanto mais precisas conforme menor for este número, fato este ressaltado por Mayhew et al. (2008), e Abadie e Wentworth (2000) em seus estudos. Brzycki (1993), ao criar uma equação de predição de 1RM baseada nas repetições até a fadiga, assinalou que sua fórmula somente é válida para predizer o 1RM quando o número de repetições até a fadiga for menor do que 10, evidência esta também observada por Reynolds et al. (2006).

O que foi encontrado neste estudo está de acordo com os autores anteriormente citados, pois em percentuais mais baixos a dispersão do número de RMs atingido foi maior (figuras 4.9 e 4.10), o que parece dificultar a precisão de uma equação para predizer o 1RM ou um percentual deste valor quando um número maior de RMs é realizado.

\section{Conclusão}

Os achados do presente estudo mostraram que a MCM, quando comparada à MCT, é aquela que melhor explica a variância de 1RM. Assim, pode ser determinado um coeficiente para a prescrição de carga de modo mais preciso baseado na MCM do que somente na MCT do indivíduo, evitando-se assim, o método de tentativa e erro para a determinação do 1RM. Os coeficientes médios gerados a partir da MCM para os exercícios supino e rosca bíceps foram 0,65 e 0,30, respectivamente. Deve-se ressaltar que estes coeficientes são específicos para o exercício e para a população nos quais eles foram elaborados.

O número de RMs no exercício monoarticular rosca scott é maior do que no exercício multiarticular supino, em todos os percentuais de 1RM avaliados.

Além disso, devido à menor dispersão do número de RMs em percentuais mais baixos de 1RM, pode-se concluir que as equações de predição são tanto mais confiáveis quanto menor for o número de repetições realizado. Assim, a utilização destas equações constitui-se em uma forma alternativa ao teste direto quando se pretende prescrever o treinamento com base na relação entre estas variáveis (\% de 1RM vs.
$\mathrm{RMs}$ ), principalmente em programas gerais de condicionamento físico.

\section{Referências}

ABADIE, B.R.; WENTWORTH, MC. Prediction of 1-RM strength from a 5-10 repetition submaximal strength test in college-aged females. Journal of Exercise Physiology; v.3, p.1-5, 2000. Disponível em:

http://faculty.css.edu/tboone2/asep/JEPonlineABA DIE.html. Acesso em: 10 set. 2010.

AMERICAN COLLEGE OF SPORTS MEDICINE (ACSM) POSITION STAND. Progression models in resistance training for healthy adults. Medicine and Science in Sports and Exercise; v.41, n.3, p.687-708, 2009. Disponível em:

http://www.ncbi.nlm.nih.gov/pubmed/19204579. Acesso em: 14 set. 2010.

BADILLO JJG, AYESTARAN EG. Fundamentos do treinamento de força: Aplicação ao alto rendimento desportivo. 2 ed. Porto Alegre: Artmed; 2007.

BAECHLE T.R., GROVES BR. Treinamento de força: passos para o sucesso. 2 ed. Porto Alegre: Artmed; 2000.

BAECHLE TR, EARLE, RW. Essentials of strength training and conditioning/National Strength and Conditioning Association. 3rd ed. Champaign, IL: Human Kinetics; 2008.

BIRD SP, TARPENNING KM, MARINO FE. Designing resistance training programmes to enhance muscular fitness: a review of the acute programme variables. Sports Medicine; v. 35 , n.10, p.841, 2005.

BRZYCKI M. Strength testing: Predicting a onerep max from reps to fatigue. Journal of Physical Education, Recreation and Dance; v.64, n.1, p.88-90, 1993.

COTTERMAN ML, DARBY LA, SKELLY WA. Comparison of muscle force production using the Smith machine and free weights for bench press and squat exercises. Journal of Strength and Conditioning Research; v.19, n.1, p.169-176, 2005.

DOURIS PC, WHITE BP, CULLEN RR, KELTZ WE, MELI J, MONDIELLO DM, et al. The relationship between maximal repetition performance and muscle fiber type as estimated by noninvasive technique in the quadriceps of untrained women. Journal of Strength and Conditioning Research; v.20, n.3, p. 699-703, 2006. 
FLECK S J, KRAEMER WJ. Fundamentos do treinamento de força muscular. 2 ed. Porto Alegre: Artmed; 2006.

FUKUNAGA T, MIYATANI M, TACHI M, KOUZAKI M, KAWAKAMI Y, KANEHISA H. Muscle volume is a major determinant of joint torque in humans. Acta Physiologica

Scandinavica; v.172, n.4, p.249-255, 2001. http://dx.doi.org/10.1046/i.1365201x.2001.00867.x

HATFIELD DL, KRAEMER WJ, SPIERING BA, HAKKINEN K, VOLEK JS, SHIMANO T, et al. The impact of velocity of movement on performance factors in resistance exercise. Journal of Strength and Conditioning Research; v.20, n.4, p.760-766, 2006.

HOEGER, WK, BARRETE, SL, HALE, DF, HOPKINS, DR. Relationship between repetitions and selected percentages of one repetition maximum. Journal of Applied Sport Science Research; v.1, n.1, p.11-13, 1987.

HOEGER, WK, HOPKINGS, DR, BARRETE, SA, HALE, DF. Relationship between repetitions and selected percentages of one repetition maximum: a comparison between untrained and trained males and females. Journal of Applied Sport Science Research; v.4, n.2, p.47-54, 1990.

JACKSON AS, POLLOCK ML, WARD A. Generalized equations for predicting body density of women. Medicine and Science in Sports and Exercise; v.12, n.3, p. 175-181, 1980.

JOHNSON MA, SIDERI G, WEIGHTMAN D, APPLETON D. A comparison of fibre size, fibre type constitution and spatial fibre type distribution in normal human muscle and in muscle from cases of spinal muscular atrophy and from other neuromuscular disorders. Journal of the Neurological Sciences; v.20, n.4, p.345-361, 1973.

KRAEMER, W.J. Exercise prescription in weight training: a needs analysis. National Strength and Conditioning Association Journal; v.5, p.64, 1983.

KRAEMER WJ. Strength training basics: Designing workouts to meet patients' goals. The Physician and Sportsmedicine; v.31, n.8, p.457, 2003. http://dx.doi.org/10.3810/psm.2003.08.457

KRAEMER WJ, HAKKINEN K. Treinamento de força para o esporte. $1^{\text {a }}$ ed. Porto Alegre: Artmed, 2004.

LOMBARDI VP. Beginning weight training: The safe and effective way. Dubuque, IA, Wm: C. Brown; 1989. http://isbndb.com/d/book/beginning weight trainin g.html. Acesso em: 14 set. 2010.

MAYHEW JL, JOHNSON BD, LAMONTE MJ, LAUBER D, KEMMLER W. Accuracy of prediction equations for determining one repetition maximum bench press in women before and after resistance training. Journal of Strength and Conditioning Research; v.22, n.5, p.1570-1577, 2008.

PEREIRA MIR, GOMES PSC, et al. Número máximo de repetições em exercícios isotônicos: influência da carga, velocidade e intervalo de recuperação entre séries. Revista Brasileira de Medicina do Esporte; v.13, n.5, p.287-291, 2007. http://www.scielo.br/pdf/rbme/v13n5/02.pdf.

Acesso em: 14 set. 2010.

http://dx.doi.org/10.1590/S151786922007000500002.

REYNOLDS JM, GORDON TJ, ROBERGS RA. Prediction of one repetition maximum strength from multiple repetition maximum testing and anthropometry. Journal of Strength and Conditioning Research; v.20, n.3, p.584-592, 2006.

SAKAMOTO A, SINCLAIR PJ. Effect of movement velocity on the relationship between training load and the number of repetitions of bench press. Journal of Strength and Conditioning Research; v.20, n.3, p.523-527, 2006.

SAÚDE OP-AD (OPAS/OMS BRASIL). Doenças crônico-degenerativas e obesidade: estratégia mundial sobre alimentação saudável, atividade física e saúde. Brasília: Organização PanAmericana da Saúde; 2003.

http://new.paho.org/bra/index.php?option=com co ntent\&task=view\&id=885\&ltemid=672. Acesso em: 12 set. 2010 .

SIRI WE. Body composition from fluid spaces and density: analysis of methods. 1961. Nutrition; v.9, n.5, p.480-91, 1993.

SPREUWENBERG LP, KRAEMER WJ, SPIERING BA, VOLEK JS, HATFIELD DL, SILVESTRE R, et al. Influence of exercise order in a resistance-training exercise session. Journal of Strength and Conditioning Research; v.20, n.1, p.141-144, 2006.

TIGGEMANN CL, KRUEL LFM, PINTO RS. A percepção de Esforço no Treinamento de Força. Revista Brasileira de Medicina do Esporte; v.16, n.4, p.301-9, 2010. http://dx.doi.org/10.1590/S151786922010000400014.

WESPTHAL M, BAPTISTA R, OLIVEIRA AR. Relações entre massa corporal total, massa 
corporal magra, área de seção transversa e $1 \mathrm{rm}$ em mulheres. Revista Brasileira de

Cineantropometria \& Desempenho Humano;

v.8, n.1, p.52-57, 2006. Disponível em:

$<$ http://www.rbcdh.ufsc.br/MostraEdicao.do?edica $\underline{0=23}$ >. Acesso em: 12 set. 2010.

WOLFE BL, LEMURA LM, COLE PJ. Quantitative analysis of single- vs. multiple-set programs in resistance training. Journal of Strength and

Conditioning Research;v.18, n.1, p.35-47, 2004.

Endereço:

Ronei Silveira Pinto

Laboratório de Pesquisa do Exercício da UFRGS

Rua Felizardo, 750

Porto Alegre RS Brasil

90690-200

Telefone: (51) 3308.5894 / Fax: (51) 3308.5843

e-mail: ronei.pinto@ufrgs.br

Recebido em: 28 de abril de 2010.

Aceito em: 11 de outubro de 2011.

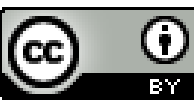

Motriz. Revista de Educação Física. UNESP, Rio Claro, SP, Brasil - elSSN: 1980-6574 - está licenciada sob Creative Commons - Atribuição 3.0 